Morphology-controlled SWCNT/polymeric microsphere arrays by a wet chemical selfassembly technique and their application for sensors

This article has been downloaded from IOPscience. Please scroll down to see the full text article.

2006 Nanotechnology 172988

(http://iopscience.iop.org/0957-4484/17/12/028)

View the table of contents for this issue, or go to the journal homepage for more

Download details:

IP Address: 143.248.224.32

The article was downloaded on 19/04/2011 at 03:33

Please note that terms and conditions apply. 


\title{
Morphology-controlled SWCNT/polymeric microsphere arrays by a wet chemical self-assembly technique and their application for sensors
}

\author{
Xing-Jiu Huang ${ }^{1,4}$, Yue $\mathbf{L i}^{2,3,4}$, Hyung-Soon $\mathrm{Im}^{1}$, Oktay Yarimaga ${ }^{1}$, \\ Ju-Hyun Kim ${ }^{1}$, Doon-Yoon Jang ${ }^{1}$, Sung-Oh $\mathrm{Cho}^{2}$, Wei-Ping Cai ${ }^{3}$ \\ and Yang-Kyu Choi ${ }^{1,5}$ \\ ${ }^{1}$ Department of Electrical Engineering and Computer Science, Korea Advanced Institute of \\ Science and Technology (KAIST), 373-1 Guseong-dong, Yuseong-gu, Daejeon, Korea \\ ${ }^{2}$ Department of Nuclear and Quantum Engineering, KAIST, 373-1 Guseong-dong, \\ Yuseong-gu, Daejeon, Korea \\ ${ }^{3}$ Key Laboratory of Material Physics, Anhui Key Lab of Nanomaterials and Nanotechnology, \\ Institute of Solid State Physics, Chinese Academy of Sciences, Hefei, 230031, Anhui, \\ People's Republic of China \\ E-mail: ykchoi@ee.kaist.ac.kr
}

Received 20 March 2006, in final form 10 April 2006

Published 2 June 2006

Online at stacks.iop.org/Nano/17/2988

\begin{abstract}
Large-scale morphology-controlled SWCNT/polymeric microsphere arrays can be obtained by a wet chemical self-assembly technique. The loading of SWCNTs, the length of SWCNTs, and the size and nature of polymeric microspheres can easily be controlled. Similar results can also be reached using this method for MWCNTs. In both types of CNTs, they form an interesting interactive 'net' structure on spheres and sphere joints. The SWCNT/PS-modified Au electrode was used for detection of uric acid by cyclic voltammetry and single-potential time-based techniques. The preliminary results show that the modified electrode presents good sensitivity and stability to uric acid.
\end{abstract}

M Supplementary data files are available from stacks.iop.org/Nano/17/2988

(Some figures in this article are in colour only in the electronic version)

\section{Introduction}

Layer-by-layer assembly is an approach based on the alternating adsorption of materials containing complementary charged or functional groups to form integrated ultra-thin films [1-5]. This method provides a powerful tool for nano- and microscale assembly of devices and novel material systems $[6,7]$ Singlewalled carbon nanotubes (SWCNTs) exhibit unique structural, mechanical, and electronic properties [8-11], and recent studies have demonstrated the use of SWCNTs in nanodevices and sensors [12-15]. Besides, a number of researchers have

\footnotetext{
4 These authors contributed equally to this work.

5 Author to whom any correspondence should be addressed.
}

shown that they can be used to form hybrid SWCNTs/polymer materials using the electrostatic layer-by-layer assembly technique [16-19]. In particular, Correa-Duarte et al deposited dense mono- and multilayers of multi-walled carbon nanotubes (MWCNTs) on silica, polystyrene, and melamine spherical colloids successfully using the polyelectrolyte-assisted layerby-layer assembly technique, and measured the conductive properties of CNT-based polymeric thin films [20, 21].

Uric acid is the principal final product of purine metabolism and is commonly found in biological fluids of humans, mainly in serum, blood, and urine. Since abnormally high levels of uric acid in the human body are a symptom of several diseases like gout, Lesch-Nyhan 
syndrome, immunodeficiency and gouty nephropathy [22], the determination of the concentration of uric acid in human blood or urine is a very important issue in diagnosing diseases.

In this paper, we present an alternative method, a wet chemical self-assembly technique [23, 24], which is based on the $\mathrm{Au}-\mathrm{S}$ chemical bonding, condensation reaction between the carboxylic termini of SWCNT and the amino group [25], alternating adsorption of monolayers of individual components attracted to each other by electrostatic and van der Waals interactions $[1-7,20,21]$, to produce large-scale SWCNT/polymeric microsphere arrays where the nanotube distribution density, length, and sphere size can be well controlled. The SWCNT/PS-modified Au electrode was used for detection of uric acid at lower concentration by cyclic voltammetry and single-potential time-based techniques. The preliminary results show that the SWCNT/PS-modified Au electrode has better electrochemical performance than that of the bare and SWCNT-modified Au electrode, which has a good potential application in biological sensors.

\section{Experimental details}

\subsection{Materials}

The SWCNTs $(1-10 \mathrm{~nm}$ in diameter, 5-20 $\mu \mathrm{m}$ in length) and MWCNTs (10-15 nm in diameter, $10-20 \mu \mathrm{m}$ in length) used in this work were purchased from Iljin Nanotech Co., Ltd (Korea), and commercialized monodispersed polystyrene spheres (PSs) of different particle sizes, $1.3 \mu \mathrm{m}$ (Soken, Chemisnow SX-130H) and $5.0 \mu \mathrm{m}$ (Soken, Chemisnow SX$350 \mathrm{H})$ in diameter, were purchased from Soken Chemical and Engineering Company. The other size PS spheres came from the Alfa Aesar Company (the standard deviation of the diameter of PSs is less than 5\%). Au (2 mm in diameter) and $\mathrm{Ag} / \mathrm{AgCl}$ electrodes were purchased from $\mathrm{CH}$ Instruments, Inc. (USA); the Pt wire electrode was obtained from Bioelectrical System, Inc. (USA). Mercaptoethylamine and uric acid were purchased from Sigma-Aldrich. Other reagents were commercially available and were of analytical grade. A phosphate-buffered saline solution (PBS, $\mathrm{pH}$ 7.4) was prepared by dissolving $1.6 \mathrm{~g} \mathrm{KCl}, 64 \mathrm{~g} \mathrm{NaCl}, 1.92 \mathrm{~g} \mathrm{KH}_{2} \mathrm{PO}_{4}$, and $11.52 \mathrm{~g} \mathrm{~K}_{2} \mathrm{HPO}_{4}$ in $800 \mathrm{ml}$ of deionized water.

\subsection{Preparation of SWCNT/polymeric composite}

$0.2 \mathrm{mg}$ raw SWCNTs and MWCNTs were chemically 'cut' by a $10 \mathrm{ml}$ mixture of concentrated sulfuric and nitric acids $(3: 1,98 \%$ and $70 \%$, respectively [25]) that were sonicated in a water bath for $8 \mathrm{~h}$ at $40^{\circ} \mathrm{C}$. The shortened SWCNTs were then collected firstly by filtering using $0.1 \mu \mathrm{m}$ nitrocellulose membranes (Advantec MFS, Inc. Dublin, CA) and washed until neutral $\mathrm{pH}$ was achieved before redispersing in $10 \mathrm{ml}$ of ethanol. The ethanol was selected as solvent here because the filter membrane can be dissolved in acetone. After the ethanol was volatilized completely, the shortened SWCNTs were redispersed in 11 acetone, hence we found the as-studied pipes can be well solubilized using a short $30 \mathrm{~min}$ sonication. This procedure yielded shortened SWCNTs with terminal carboxylate groups. The simple preparation procedure of $2 \mathrm{mg}^{-1}$ SWCNT solution was as follows: taking $100 \mathrm{ml}$ $0.2 \mathrm{mg}^{-1}$ SWCNT/acetone solution and then evaporating
$90 \mathrm{ml}$ acetone at room temperature. It is worth pointing out here that the concentration is only an approximate value, which has no obvious effect on our experimental results (considering the loss during the experiment).

A gold surface of polymeric sphere ordered arrays was obtained by sputtering with $30 \mathrm{~nm} \mathrm{Au} \mathrm{(99.99 \% )} \mathrm{firstly.}$ $100 \mu 1$ mercaptoethylamine solution was then dropped onto the array surface, followed by exhaustive washing using double deionized water and acetone $6 \mathrm{~h}$ later and then dried at room temperature. The mercaptoethylamine monolayer on the polymeric microsphere surface was formed and amino groups were introduced to the surface.

CNT solution was dropped onto the mercaptoethylamine modified polymeric sphere surface, and then the chemically shortened SWCNTs accompanied with carboxyl groups at the open ends reacted with the amino groups on the sphere surface. In the experiment, the deposition cycle is defined as the process performed from dropping the first SWCNT solution to the second one.

\subsection{Electrochemical measurements}

The as-prepared SWCNT solution was dropped onto the mercaptoethylamine modified gold electrode surface to give an SWCNT-modified Au electrode. The detailed information of SWCNT/PS modified electrode preparation can be obtained from section 2.2. Cyclic voltammetry (CV) measurements were performed on a BAS $100 \mathrm{~W}$ electrochemical analyser (Bioanalytical Systems, Inc., USA). All CV experiments were carried out in PBS solution in a conventional three-electrode cell at room temperature. $\mathrm{An} \mathrm{Ag} / \mathrm{AgCl}$ and a $\mathrm{Pt}$ wire were employed as reference and counter-electrodes, respectively.

\subsection{Characterization}

Atomic force microscopy (AFM) was done with a Nanoscope III (Digital Instruments, Veeco Metrology, LLC, CA) scanning probe microscope. The FTIR spectrum was performed using a model MB-154 analyser (Bomem Inc., CA). All of the SEM images were obtained with a Philips XL 30 AFEG scanning electron microscope (Eindhoven, The Netherlands) except image (d) in figure 4 (Hitachi S-4800 scanning electron microscope, Japan).

\section{Results and discussion}

\subsection{Properties of shortened carbon nanotubes and composite pattern}

The mixture of concentrated sulfuric and nitric acids with volume ratio of $3: 1$ was chosen here as an effective method for cutting the raw SWCNTs. This procedure introduces carboxylic acid functionalities at the ends of the CNTs as well as some carboxylic acid units at the CNT sidewalls $[24,26]$. In order to demonstrate the presence of carboxyl groups on our mixture of acid-treated SWNTs, FTIR spectrum measurement was conducted. The result inset in figure 1(b) strongly suggests that the carbon nanotubes are open and terminated with carboxylic acid groups $(-\mathrm{COOH}$, IR stretching vibration frequency $v_{\mathrm{C}=\mathrm{O}}=1638 \mathrm{~cm}^{-1}$ ) and carboxylate groups $\left(-\mathrm{COO}^{-}, v_{\mathrm{C}=\mathrm{O}}=1530 \mathrm{~cm}^{-1}\right)$ after oxidation treatment. The shortened carbon nanotubes were 


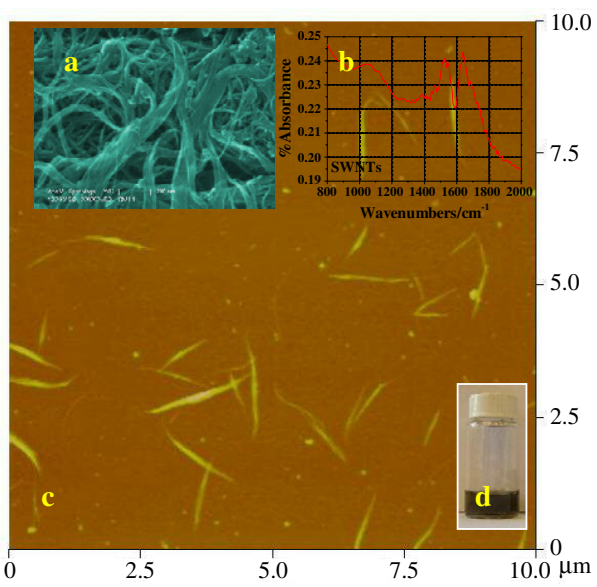

Figure 1. (a) SEM image of raw SWCNTs. (b) FTIR spectrum of cut SWCNTs. (c) Typical AFM image of dispersed cut SWCNTs (after sonication for $8 \mathrm{~h}$ at $40{ }^{\circ} \mathrm{C}$ ). (d) The picture of well solubilized cut SWCNT/acetone solution.

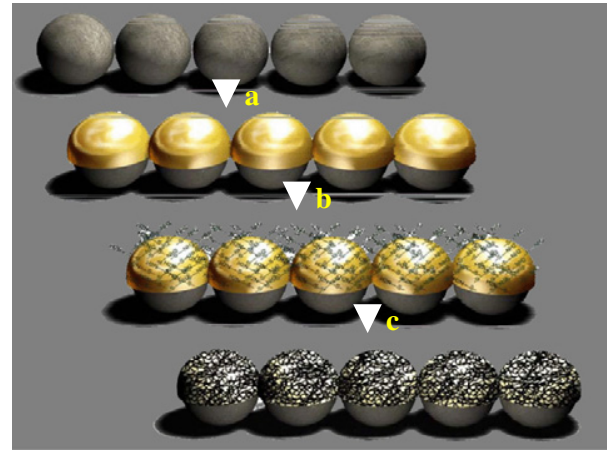

Figure 2. Illustration for the preparation of CNT/polymeric sphere arrays: (a) polymeric sphere arrays were fabricated and then coated with a thin gold layer; (b) mercaptoethylamine-modified polymeric spheres; (c) wet chemical self-assembly of CNTs onto the surface of sphere arrays.

characterized by AFM (as shown in figure 1(c)). The images indicate that the morphology of the SWCNT bundles changed from that of highly entangled strands (see figure 1(a)) to flexible-rod-like ones which were thoroughly dispersed in acetone after etching. The length of the shortened carbon nanotubes is about $0.5-1.5 \mu \mathrm{m}$. The flexibility of the SWCNTs [27-29], taken together with the carboxylic group located at the end and sidewall of SWCNTs, allows them to bond fairly small particles.

Patterning the resulting ordered arrays with a continuous and homogeneous distribution of carbon nanotubes and conductive properties can be achieved, as outlined in figure 2 . Firstly, the colloid monolayer of polymeric spheres was fabricated on the substrate and then was coated with a $30 \mathrm{~nm}$ thick gold layer; subsequently, it was modified by mercaptoethylamine to introduce the amino group. Lastly, carboxylated SWCNT solution was deposited onto the surface in a controllable way.

\subsection{Assembly at different SWCNT concentrations}

The monolayer arrays of polystyrene (PS) microspheres with large area around $\mathrm{cm}^{2}$ in size were fabricated by the spin

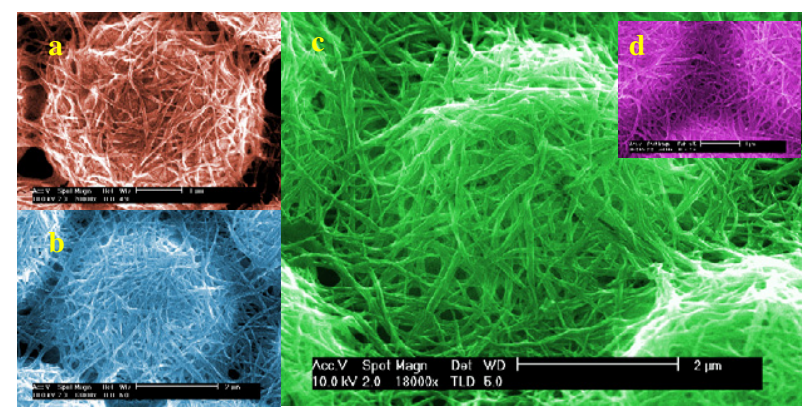

Figure 3. SEM images of SWCNT/PS microspheres under one deposition cycle at different SWCNT concentrations. Images (a) and (b) correspond to the thin- and multi-layer SWCNTs, respectively, but image (c) shows multi-layer SWCNTs have been obtained with a tilting angle of $40^{\circ}$. Image (d) shows the 'net' structure on spheres and sphere joints from the top view. Concentrations in (b), (c), and (d) are $2 \mathrm{mg} \mathrm{l}^{-1}$ and that in (a) is $0.2 \mathrm{mg} \mathrm{l}^{-1}$.

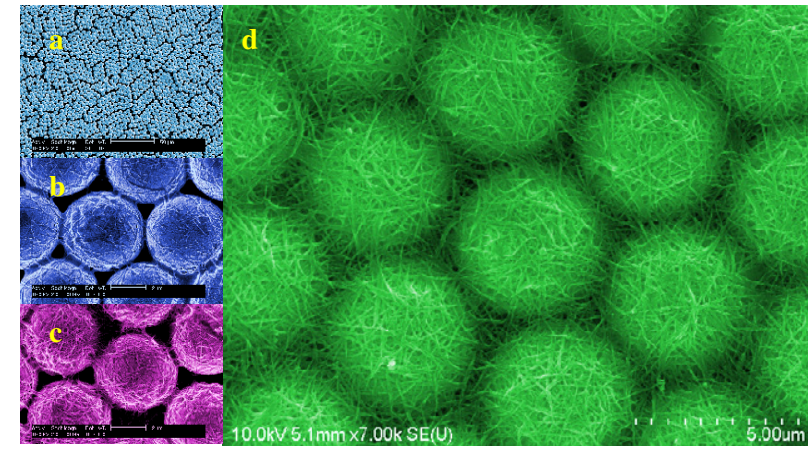

Figure 4. Typical SEM images of different layers of SWCNTs on PS microsphere arrays under different deposition cycles at the given concentration of $0.2 \mathrm{mg}^{-1}$. Image (a) shows the large-scale morphology-controlled SWCNT/PS microsphere arrays ( 20 cycles), and images (b), (c), and (d) for the different numbers of SWCNT layers on arrays corresponding to one cycle, five cycles, and 20 cycles, respectively.

coating method on the silicon wafer and then heated at $130{ }^{\circ} \mathrm{C}$ for $40 \mathrm{~min}$ in order to guarantee the hard adherence of these arrays to the substrate as we previously reported [30-33]. This colloid monolayer was used as a substrate for the assembly of mono- and multi-layer SWCNTs, which provides a large specific surface area. The PS sphere surface was coated with a $30 \mathrm{~nm}$ gold layer firstly in a clean room, and then functionalized with a primary monolayer of mercaptoethylamine. Finally, the SWCNTs were assembled on the PS sphere surface by the covalent bond of $-\mathrm{NH}-\mathrm{CO}-$.

Figure 3 shows the typical SEM images of microspheres with diameter of $5 \mu \mathrm{m}$ after one deposition cycle under 0.2 and $2 \mathrm{mg} \mathrm{l}^{-1}$ SWCNT concentrations. It is obvious from the figure that SWCNTs can be assembled on the polymeric microsphere surface continuously and homogeneously by controlling the concentration at the deposition step. Firstly, SWCNTs are assembled on the 2D microsphere surface easily due to the condensation reaction between the $-\mathrm{COOH}$ and $-\mathrm{NH}_{2}$; this step is different from physical adsorption, as evidenced by the stability of the layers when ultrasonicated. Owing to the chemical bonds, a few SWCNTs are chemisorbed onto the mercaptoethylamine-modified PS microsphere surface, 
adapting themselves to the morphology of the interdigitated thin layer which is different from the multi-layer in the thickness (see figure 3(a)). The elasticity of CNTs has been reported recently, and the existence of the bending mechanism was demonstrated by predominantly tethering the CNTs to the functionalized surface through their carboxyl end groups [21]. With increasing SWCNT concentration, many SWCNTs were sequentially adsorbed onto the sphere surface to form an interdigitated multi-layer because of electrostatic and van der Waals interactions and formed 3D structures (see figures 3(b) and (c)). As can be seen from the SEM images, carbon nanotubes distribute on the surface uniformly and serve as connecting lines between neighbouring spheres within the resulting composite of thin and multi-layers. In addition, the SWCNTs form an interesting interactive 'net' structure on spheres and sphere joints (see figure 3(d)), which can make a structure with good conductivity for future applications of electrochemical sensors and electronic devices.

\subsection{Assembly at different deposition cycles}

In our experiments, we can control not only the amount of SWCNTs (different SWCNT concentration for each deposition cycle) but also different deposition cycles at a given concentration to obtain SWCNT/PS microsphere arrays. Figure 4 shows SEM images for the SWCNT layers on the monolayer PS microsphere arrays under different deposition cycles (one cycle for image (b), five cycles for image (c), and 20 cycles for images (a) and (d)) at the given concentration of $0.2 \mathrm{mg}^{-1}$. Obviously, carboxylated carbon nanotubes can be assembled on the surface of micro-polymeric sphere arrays uniformly by controlling the deposition cycles at the given concentration. It is better to point out two important structural characteristics here. First of all SWCNTs uniformly cover the entire surface of the PS spheres without phase separation, and secondly the surface packing layers of the shortened SWCNTs increased rapidly with increasing deposition cycles. This also indicates that a steady SWCNT layer can be obtained by controlling the deposition cycles. Figure 4 shows an interdigitated layer of SWCNT which is deposited in each deposition cycle due to the alternating adsorption of anionic and cationic bipolar components onto PS surfaces via the electrostatic attraction and van der Waals interactions. The final morphology of the multi-layers can be described as a mixture of shortened carbon nanotubes intricately interwoven together on the PS microsphere arrays (see figure 4(d)). We suggest that the increase in the surface packing layers with the deposition cycles was achieved by changing the acting force from the $\mathrm{Au}-\mathrm{S}$ covalent bond to electrostatic and van der Waals interactions between the carbon nanotubes. Therefore, the wet chemical self-assembling technique should, in principle and in experiments, enable us to prepare uniformly dispersed SWCNT/PS microsphere arrays with a controllable packing density by simply regulating the number of deposition cycles or relative amounts of the cut SWCNT components within each of the constituent layers. Apparently, the assembly of SWCNTs with different lengths onto microsphere surfaces of various sizes and natures as templates can also be realized using this method (supplementary information is available at stacks.iop.org/Nano/17/2988).

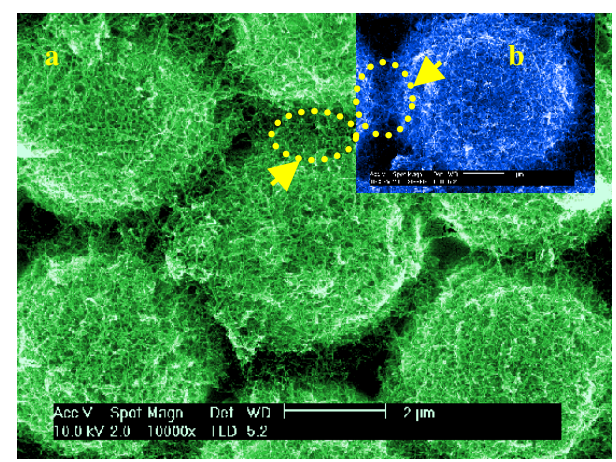

Figure 5. SEM images of MWCNTs assembled on PS sphere surface. Image (a) is for MWCNTs/PS ordered arrays, whereas image (b) corresponds to multilayer MWCNTs on an individual sphere. Concentration, $0.2 \mathrm{mg} \mathrm{l}^{-1}$; deposition cycles, 20.

The wet chemical self-assembling technique can also be used here to assemble multi-walled carbon nanotubes (MWCNTs) on the monolayer PS microsphere arrays. Similarly, prior to assembly, raw MWCNTs were oxidized by an acid mixture to form carboxylated carbon nanotubes (see section 2 for the detailed procedure). The MWCNT layers can easily be controlled by the deposition cycles or MWCNT concentration. Certainly, MWCNTs can also be assembled on any other micro- or nano-spheres with different size. Figure 5 shows the SEM results of assembling MWCNTs on the PS microsphere arrays under 20 deposition cycles at the concentration of $0.2 \mathrm{mg} \mathrm{l}^{-1}$. Obviously, PS spheres are connected by 3D MWCNT 'net' structures, indicated by yellow arrows.

\subsection{Preliminary electrochemical behaviour}

The redox reactions of potassium ferrocyanide usually serve as a benchmark in investigating electrochemistry at different carbon electrodes [34]. Here, $\mathrm{Fe}(\mathrm{CN})_{6}^{3-} / \mathrm{Fe}(\mathrm{CN})_{6}^{4-}$ as redox pairs were used to investigate the electrochemical performance and electron transfer properties of the SWCNT/PS electrode. Figure 6(a) indicates the potential window of the bare $\mathrm{Au}$ electrode, SWCNT-modified Au electrode, and SWCNT/PS electrode for $\mathrm{K}_{3} \mathrm{Fe}(\mathrm{CN})_{6}$ in $0.1 \mathrm{~mol}^{-1} \mathrm{PBS}$. Firstly, it is found that the current separation between the forward and backward scans becomes greater that that of the bare gold electrode and the SWCNT modified gold electrode. This agrees with the large capacitance feature reported before $[35,36]$. The capacitance is calculated from the $\mathrm{CV}$ curves, with $C=i / v$, where $i$ is the current and $v$ the scan rate $\left(\mathrm{V} \mathrm{s}^{-1}\right)$. At the potential of $-0.05 \mathrm{~V}$, for example, the effective capacitance is about two orders of magnitude higher than that of the SWCNTs, and over three orders of magnitude higher than the conventional bare gold electrode. This high capacitance is consistent with the large surface area of the SWCNT/PS composite [36, 37]. Secondly, the voltammetric peak at the SWCNT/PS electrode is broad, suggesting slow electron transfer kinetics, presumably due to the macroscopic plane-like structure. Finally, we observed that the SWCNT/PS electrode has a good stability; the result is shown in figure 6(b). This is because SWCNT assembly on the substrate is via the Au$\mathrm{S}$ chemical bond, electrostatic and van der Waals interactions. 

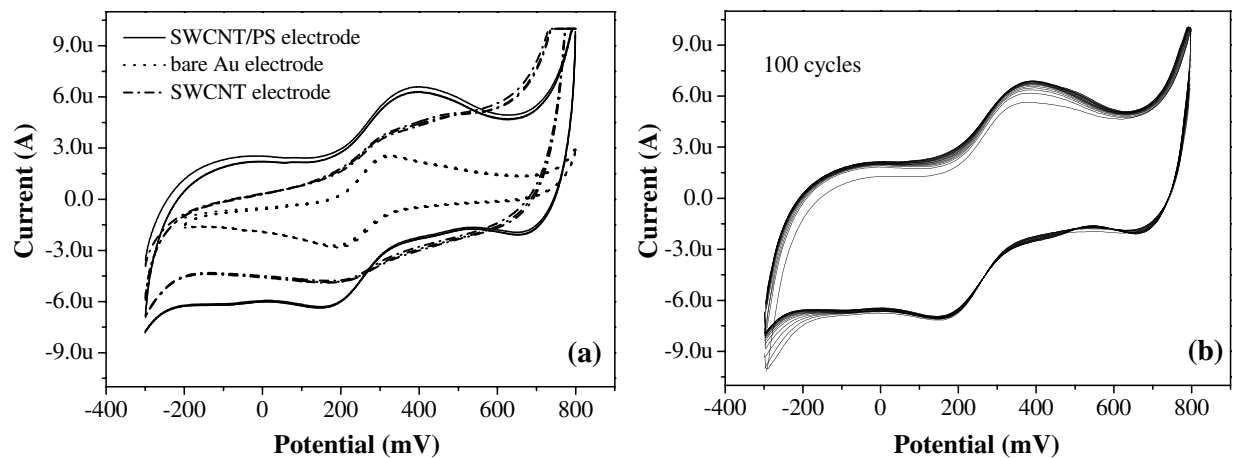

Figure 6. (a) Potential windows for bare gold, SWCNT, and SWCNT/PS electrodes in $5 \times 10^{-4} \mathrm{~mol}^{-1} \mathrm{~K}_{3} \mathrm{Fe}(\mathrm{CN})_{6} / \mathrm{PBS}$; (b) the stability of as-studied SWCNT/PS electrode, $5 \times 10^{-4} \mathrm{~mol} \mathrm{l}^{-1} \mathrm{~K}_{3} \mathrm{Fe}(\mathrm{CN})_{6} / \mathrm{PBS}$; scan rate of $100 \mathrm{mV} \mathrm{s}^{-1}$.
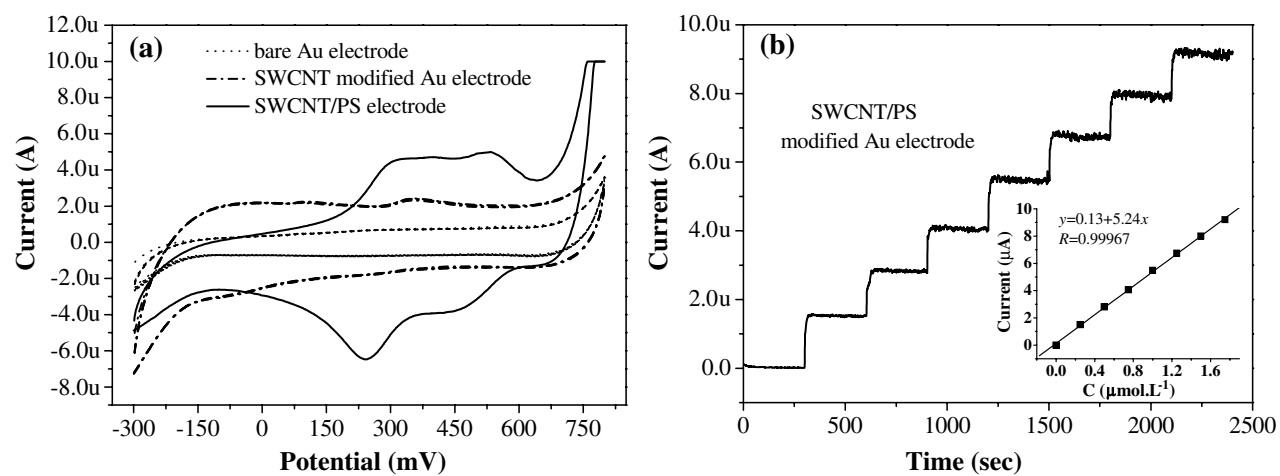

Figure 7. (a) Cyclic voltammograms of bare gold, SWCNT-modified, and SWCNT/PS-modified Au electrodes in $1 \times 10^{-6} \mathrm{~mol}^{-1}$ uric acid/PBS solution at a scan rate of $100 \mathrm{mV} \mathrm{s}^{-1}$; (b) real-time detection of the current for the SWCNT/PS-modified Au electrode in uric acid solution for concentrations from $2.5 \times 10^{-7}$ to $1.75 \times 10^{-6} \mathrm{~mol} \mathrm{l}^{-1}$ (each addition $2.5 \times 10^{-7} \mathrm{~mol} \mathrm{l}^{-1}$ ); scan rate, $100 \mathrm{mV} \mathrm{s}^{-1}$; initial potential, $320 \mathrm{mV}$. The inset shows a plot of the current versus concentration; the black square points are experimental data, and the line is a linear fit through these data.

Therefore, we suggest that the electrochemical behaviour was improved by our present effort on SWCNT/PS introduction. Such advantages enable us to study its application for biosensors such as uric acid, aspartate aminotransferase (GOT) and alanine aminotransferase (GPT) detection, etc.

Figure 7(a) indicates the comparison of bare gold, SWCNT-modified, and SWCNT/PS-modified Au electrodes in $1 \times 10^{-6} \mathrm{~mol}^{-1}$ uric acid/PBS solution at a scan rate of $100 \mathrm{mV} \mathrm{s}^{-1}$. Firstly, no current peak for uric acid can be observed at the bare gold electrode, suggesting that it is hard for uric acid to accumulate at the gold electrode surface and/or the electrochemical reaction is very slow. Secondly, at the SWCNT-modified Au electrode, there is only a weak and broad oxidation peak around $360 \mathrm{mV}$. Finally, at the SWCNT/PS modified Au electrode, two anodic peaks occur at about 320 and $530 \mathrm{mV}$, and two cathodic peaks occur at around 240 and $460 \mathrm{mV}$. It can be inferred that uric acid can effectively accumulate at the SWCNT/PS electrode surface and that its electrochemical oxidation was promoted. This is likely due to the fact that the as-studied SWCNT/PS composite has an interesting interactive 'net' structure on spheres and sphere joints; on the other hand, the acid treatment creates more oxide defects on the tube sidewalls [38] and ends [38-40], which can be involved in the redox reaction and make the SWCNT more hydrophilic so that the sample can contact the surface better.
Figure 7(b) shows the measurement of current as a function of time and solution concentration at the SWCNT/PS modified Au electrode. It is obvious that the current increases stepwise with discrete changes in concentration of uric acid from $2.5 \times 10^{-7}$ to $1.75 \times 10^{-6} \mathrm{~mol}^{-1}$ and that the current is constant for a given concentration; the response is very fast in reaching a dynamic equilibrium upon each addition of the sample solution, generating a steady-state current signal within several seconds; the changes in current are also reversible for increasing and/or decreasing uric acid concentration. A typical plot of the peak current versus concentration (the inset in figure 7(b)) shows that this concentration dependence is linear over concentration from $2.5 \times 10^{-7}$ to $1.75 \times 10^{-6} \mathrm{~mol}^{-1}$.

\section{Conclusions}

In summary, the results reported here demonstrate that largescale morphology-controlled SWCNT/polymeric microsphere arrays can be obtained by a wet chemical self-assembly technique. The loading of SWCNTs can be easily controlled by the deposition cycles or relative amounts of the cut SWCNT components within each of the constituent layers and the length of the studied SWCNTs can be controlled by the oxidization conditions such as temperature, the amount of reactant (tubes and acid), sonication time, etc. On the other hand, the morphology of the composite can also be controlled 
by using arrays of microspheres with different sizes. Similar results can be reached by using the wet chemical method stated above as for MWCNTs. Besides, both types of CNTs form an interesting interactive 'net' structure on spheres and sphere joints. Preliminary electrochemical results show that the SWCNT/polymeric microsphere array electrode has better CV performance and stability. The SWCNT/PS-modified Au electrode exhibits stronger electrochemical activity to uric acid than that of bare gold and SWCNT-modified electrodes even at lower concentration $1 \times 10^{-6} \mathrm{~mol}^{-1}$. In addition, a good linear relationship between current and concentration was obtained from $2.5 \times 10^{-7}$ to $1.75 \times 10^{-6} \mathrm{~mol}^{-1}$. Future studies will investigate the detailed properties of biosensors such as selectivity, effect of $\mathrm{pH}$, effect scan rate, electron transfer mechanism, etc.

\section{Acknowledgments}

This work was supported by the Brain Korea 21 project, the School of Information Technology, the Korea Advanced Institute of Science and Technology in 2006, and the National Research and Development Program (NRDP, 2005-01274) for the biomedical function monitoring biosensor development sponsored by the Korea Ministry of Science and Technology (MOST).

\section{References}

[1] Hammond P T 2004 Adv. Mater. 151271

[2] Liu J W, Li X J, Schrand A, Ohashi T and Dai L M 2005 Chem. Mater. 176599

[3] Olek M, Ostrander J, Jurga S, Möhwald H, Kotov N, Kempa K and Giersig M 2004 Nano Lett. 41889

[4] Mamedov A, Ostrander J, Aliev F and Kotov N A 2000 Langmuir 163941

[5] Correa-Duarte M A, Perez-Juste J, Sanchez-Iglesias A, Giersig M and Liz-Marźn L M 2005 Angew. Chem. Int. Edn 444375

[6] Bruening M L and Sullivan D M 2002 Chem. Eur. J. 83832

[7] Caruso F, Spasova M, Susha A, Giersig M and Caruso R A 2001 Chem. Mater. 13109

[8] Xiao Y, Patolsky F, Katz E, Hainfeld J F and Willner I 2003 Science 2991877

[9] Dai L and Mau A W H 2001 Adv. Mater. 13899

[10] Xie S S, Chang B H, Li W Z, Pan Z W, Sun L F, Mao J M, Chen X H, Qian L X and Zhou W Y 1999 Adv. Mater. 111135

[11] Shimoda H, Gao B, Tang X P, Kleinhammes A, Fleming L, Wu Y and Zhou O 2002 Phys. Rev. Lett. 8815502
[12] Cao A, Talapatra S, Choi Y Y, Vajtai R, Ajayan P M, Filin A, Persans P and Rubio A 2005 Adv. Mater. 17147

[13] Barone P W, Baik S, Heller D A and Strano M S 2005 Nat. Mater. 486

[14] Santhanam K S V, Sangoi R and Fuller L 2005 Sensors Actuators B 106766

[15] Staii C and Johnson A T 2005 Nano Lett. 51774

[16] Artyukhin A B, Bakajin O, Stroeve P and Noy A 2004 Langmuir 201442

[17] Mamedov A A, Kotov N A, Prato M, Guldi D M, Wicksted J P and Hirsch A 2002 Nat. Mater. 1190

[18] Qin S H, Qin D Q, Ford W T, Zhang Y J and Kotov N A 2005 Chem. Mater. 172131

[19] Paloniemi H, Lukkarinen M, Aaritalo T, Areva S, Leiro J, Heinonen M, Haapakka K and Lukkari J 2006 Langmuir 2274

[20] Correa-Duarte M A, Kosiorek A, Kandulski W, Giersig M and Liz-Marzán L M 2005 Chem. Mater. 173268

[21] Correa-Duarte M A, Kosiorek A, Kandulski W, Giersig M and Salgueiriño-Maceira V 2006 Small 2220

[22] Dutt V S E and Mottola H A 1974 Anal. Chem. 461777

[23] Liu Z F, Shen Z Y, Zhu T, Hou S F and Ying L Z 2000 Langmuir 163569

[24] Wu B, Zhang J, Wei Z, Cai S M and Liu Z F 2001 J. Phys. Chem. B $\mathbf{1 0 5} 5075$

[25] Liu J et al 1998 Science 2801253

[26] Sheeney-Haj-Ichia L, Basnar B and Willner I 2005 Angew. Chem. Int. Edn $\mathbf{4 4} 78$

[27] Ko H, Peleshanko S and Tsukruk V V 2004 J. Phys. Chem. B 1084385

[28] Wong E W, Sheehan P E and Lieber C M 1997 Science 2771971

[29] Shimoda H, Oh S J, Geng H Z, Walker R J, Zhang X B, McNeil L E and Zhou O 2002 Adv. Mater. 14899

[30] Li Y, Cai W P, Duan G T, Cao B Q and Sun F Q 2005 J. Mater. Res. 20338

[31] Li Y, Cai W P, Cao B Q, Duan G T, Li C C, Sun F Q and Zeng H B 2006 J. Mater. Chem. 16609

[32] Li Y, Cai W P, Cao B Q, Duan G T, Sun F Q, Li C C and Jia L C 2006 Nanotechnology 17238

[33] Sun F Q, Cai W P, Li Y, Cao B Q, Lu F, Duan G T and Zhang L D 2004 Adv. Mater. 161116

[34] Bard A J and Faulkner L R 2001 Electrochemical Methods: Fundamentals and Applications (New York: Wiley)

[35] Barisci J N, Wallace G G and Baughman R H 2000 J. Electrochem. Soc. 1474580

[36] Du C S, Yeh J and Pan N 2004 Nanotechnology 16350

[37] Liu C Y, Bard A J, Wudl F, Weitz I and Heath J R 1999 Electrochem. Solid-State Lett. 2577

[38] Banks C E, Davies T J, Wildgoose G G and Compton R G 2005 Chem. Commun. 7829

[39] Banks C E, Moore R R, Davies T J and Compton R G 2004 Chem. Commun. 161804

[40] Chou A, Böcking T, Singh N K and Gooding J J 2005 Chem. Commun. 7842 